\title{
Gastronomie und Kulinarik
}

\author{
Coordinating Lead Authors (CLAs) \\ Dagmar Lund-Durlacher \\ Lead Authors (LAs) \\ Dagmar Lund-Durlacher, Stefan Gössling, Hannes Antonschmidt, \\ Gudrun Obersteiner, Egon Smeral \\ Contributing Authors (CAs) \\ Martin Wildenberg
}

Die Gastronomie ist einerseits ein wichtiger Abnehmer der Landwirtschaft und der Nahrungsmittelindustrie, anderseits liefert sie wichtige Serviceleistungen an Touristen (touristischer Konsum von In- und Ausländern) und die lokale Bevölkerung (privater Konsum) sowie an aus- und inländische Dienst- und Geschäftsreisende. Gerade für Touristen stellt das Speise- und Getränkeangebot ein wichtiges Element nahezu jeder Urlaubsreise dar. Für Hotel- und Gastronomiebetriebe ist es daher wichtig, ein exzellentes Speisen- und Getränkeangebot zu gestalten, das auch die für den Gast wichtigen Nachhaltigkeitsaspekte v. a. auch in Hinblick auf den Klimawandel berücksichtigt (Lund-Durlacher et al. 2016). Insgesamt bieten sich den Hotel- und Gastronomiebetrieben einige Handlungsoptionen hinsichtlich einer klimaschonenden Angebotsgestaltung, die in diesem Kapitel detailliert abgehandelt werden.

\subsection{Allgemeine Entwicklung und Trends}

Mehrere generelle Trends kennzeichnen die globale Nahrungsmittelsituation. Dies ist einerseits die mit etwa 83 Mio. Menschen pro Jahr wachsende Weltbevölkerung und die damit verbundene Steigerung der Nahrungsmittelnachfrage (UN 2017), zum anderen der steigende Konsum energieintensiver und treibhausgasemittierender Nahrungsmittel, d. h. tierischen Proteins (McMichael et al. 2007; Kearney 2010). Aktuell hungern noch immer viele Menschen in Ländern Afrikas, Indiens und Südostasiens; gleichzeitig wird davon ausgegangen, dass sich die Ernte wichtiger Grundnahrungsmittel (Getreide, Mais, Soja und Reis) bis zum Jahr 2050 aufgrund des Klimawandels in fast allen Teilen der Welt reduzieren wird (Wheeler und von Braun 2013). Bekannt ist auch, dass große Lebensmittelmengen vorrangig in den Industrienationen verschwendet werden: Die Food and Agriculture Organisation (FAO et al. 2013) geht weltweit von 1,3 Mrd. Tonnen jährlich aus.
Den Gastronomiebetrieben kommt eine besondere Bedeutung beim Nahrungsmittelkonsum zu, weil immer mehr Menschen im Tourismus, in der Freizeit, aber auch bei der Arbeit außer Haus Essen gehen. Zur Foodservice-Industrie gehören Restaurants, Bars und Cafés, Schnellimbisse, Lieferküchen und Partyservice (Catering), Kantinen (z. B. in Schulen, Betrieben) sowie Kioske (z. B. Würstelstände) und ambulante Angebote (z. B. Foodtrucks). Eine globale Schätzung für den Tourismus geht davon aus, dass im Jahr 2005 mindestens 75 Mrd. Mahlzeiten in gastronomischen Betrieben konsumiert wurden, 200 Mio. am Tag (Gössling et al. 2011). Dazu kommen Essen bei der Arbeit sowie jenes einheimischer Restaurantbesucher. Mit dem erwarteten globalen Zuwachs im Tourismus - die Zahl der internationalen Ankünfte wuchs um 7 \% im Jahr 2017 (UNWTO 2018) kommt der Gastronomie eine global immer größere Rolle bei der Nahrungsmittelversorgung zu, auch weil die Restaurantbesuche der lokalen Bevölkerung wachsen.

Dies ist auch in Österreich der Fall. Grob geschätzt werden bei 110,4 Mio. Übernachtungen ausländischer Touristen sowie 39,4 Mio. Übernachtungen inländischer Touristen (Statistik Austria 2019a), die mindestens drei Mal am Tag eine Mahlzeit zu sich nehmen, rund 450 Mio. Essen allein im Tourismus Österreichs konsumiert (im Jahr 2018). Dazu kommen die Mahlzeiten von Tagestouristen und Ausflugsgästen, Restaurantbesuche der Österreicher im Alltag, Gemeinschaftsverpflegung (in Großunternehmen, Schulen, Krankenhäusern u. a.) sowie die Belieferung von Feiern und Veranstaltungen.

Internationale Studien zeigen, dass das Essen in Gastronomiebetrieben für den Klimawandel von besonderer Bedeutung ist, weil diese in aller Regel bestrebt sind, Grundzutaten möglichst preisgünstig einzukaufen. Dies bedeutet häufig, dass große Mengen an Nahrungsmitteln aus der Massenproduktion eingekauft und über große Distanzen transportiert werden. Dazu kommt, dass insbesondere Hotels häufig ihren 


\section{Durchschnittlicher steuerbarer Umsatz 2016 je Einwohner in der Wirtschaftsabteilung „Gastronomie“ nach politischen Bezirken}

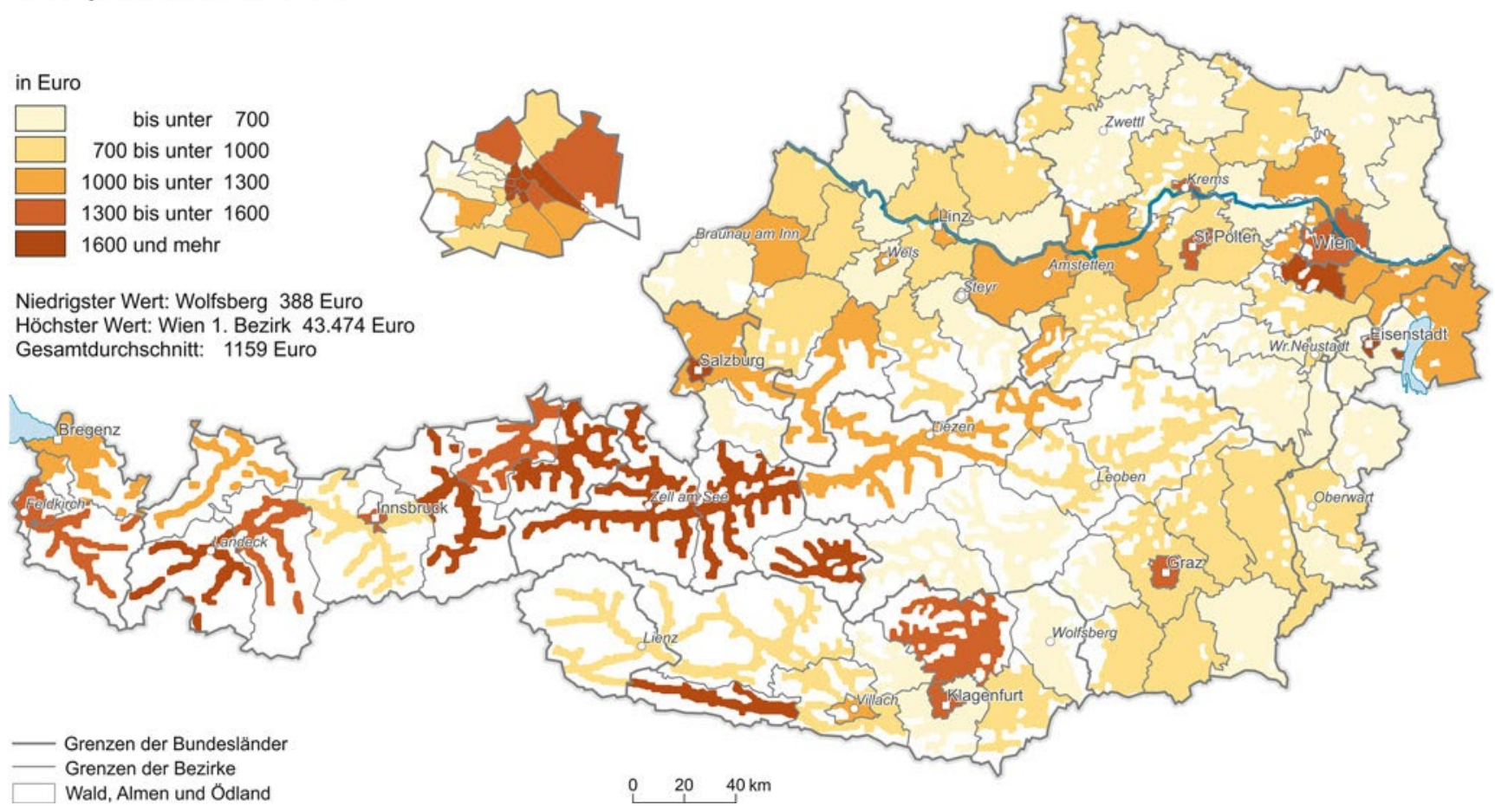

Abb. 5.1 Durchschnittlich steuerbarer Umsatz 2016 je Einwohner in der Wirtschaftsabteilung „Gastronomie“ nach politischen Bezirken. (Statistik Austria 2019b)

Gästen Essen mit hohem Proteinanteil anbieten (in der Regel Fleisch) und in der Gastronomie mehr Essen weggeworfen wird als zu Hause (Gössling et al. 2011; Juvan et al. 2018).

\subsubsection{Marktentwicklung und ökonomische Bedeutung der Gastronomiebetriebe}

Im Jahr 2019 waren in Österreich 39.180 Gastronomiebetriebe registriert, was einer weiteren leichten Abnahme der Zahl der Betriebe entspricht (seit 2010: -3,8 \%; WKO 2020). Kleinbetriebe (mit bis zu 9 Beschäftigten) dominieren (92\%), größere Gastronomiebetriebe mit 50 und mehr Beschäftigten machen nur $0,7 \%$ der Gesamtzahl aus (WKO 2020). Insgesamt erzielten die Gastronomiebetriebe im Jahr 2017 Umsatzerlöse von rund 10,19 Mrd. $€$. Die pro Einwohner höchsten Gastronomieumsätze werden in den urbanen Ballungsräumen (an der Spitze Wien) und in Tourismusdestinationen generiert (Abb. 5.1). Investitionen wurden in Höhe von 439 Mio. $€$ getätigt (WKO 2020).

Die touristischen Gesamtaufwendungen in- und ausländischer Österreich-Gäste lassen sich im Rahmen des Tourismussatellitenkontos (TSA) nach Ausgabenkategorien unterteilen. Die Detailgliederung weist den Bereich Gastronomie im Berichtsjahr 2017 mit 25,9\% des touristischen Gesamtkonsums neben der Beherbergung (32\%) als einen der mit Abstand bedeutendsten Bereiche aus. Neben der Beherbergung $(+4,4 \%)$ konnte auch die Gastronomie ihr Gewicht aufgrund einer überdurchschnittlichen Wachstumsrate von $+4,2 \%$ gegenüber 2016 leicht ausbauen (Fritz et al. 2019).

\subsubsection{Angebots- und Nachfragetrends (national, international)}

Für Österreich-Urlauber spielt das Thema Kulinarik eine große Rolle. Zwar haben im Tourismusjahr 2017/2018 nur rund $5 \%$ der Urlaubsgäste $(+3 \%$-Punkte gegenüber 2013/2014) eine explizit kulinarische Reise nach Österreich unternommen (Kulinarik als Hauptmotiv), doch kann in diesem Zusammenhang auch festgestellt werden, dass die Gäste im Winter bei den sonstigen, nichtsportlichen Aktivitäten an erster Stelle „,ins Kaffeehaus gehen“ (54 \% der Wintergäste in Städten) und an zweiter Stelle „Essen gehen (außerhalb der Unterkunft)“ (47 \%) nennen. „Typische Speisen/Getränke der Region genießen" nimmt mit $32 \%$ den fünften Platz ein (Österreich Werbung 2019, S. 23). Der Sommer zeigt ein ähnliches Bild. $51 \%$ der Gäste in Städten gaben an ,ins Kaffeehaus zu gehen" (dritter Platz unter den sonstigen Aktivitäten), 51 \% der Österreich-Gäste nennen „Essen gehen (außerhalb der Unterkunft)“ und $39 \%$,typische Speisen/Getränke der Region genießen“ (Österreich Werbung 2019; vgl. 
auch Abschn. 5.2). Eine Kurzcharakteristik des Kulinarikurlaubers zeigt, dass häufig der Partner oder die Partnerin mit auf der Reise ist (45\%), der Großteil aus Österreich (39\%) und Deutschland (30\%) kommt und das Durchschnittsalter bei 47,6 Jahren liegt. Neben dem ,regionalen Speisen- und Getränkeangebot" (53 \%) entscheidet sich der Kulinarikgast auch aufgrund von „Ortsbild, Architektur, Bauwerke“ (27\%), „Landschaft, Natur“ (25\%), „Sehenswürdigkeiten, Ausflugsziele“ (23\%) sowie ,gute Luft, Klima“ (21\%) für die Reise nach Österreich (Österreich Werbung 2019).

Bezüglich der Ernährungsgewohnheiten im Kontext der Klimawandeldiskussion ist ein Trend bemerkenswert: der Trend zur Reduzierung des Fleischkonsums. Rund $10 \%$ der Österreicher ernähren sich fleischlos, jeder vierte Österreicher gilt als „Flexitarier“ und reduziert den Fleischkonsum bewusst (Meinungsraum 2018). Im wichtigsten Herkunftsmarkt Deutschland wird der Anteil der Vegetarier und Veganer auf rund $6 \%$ geschätzt (ProVeg International 2019), mit steigender Tendenz in beiden Ländern. Da die fleischlose Ernährung in den letzten Jahren zu den gesellschaftlichen Trends zählt, sind Fleischersatzprodukte auch in der Gastronomie immer gefragter und viele Betriebe stellen sich auf diese Nachfrage ein. Laut einer Erhebung von ProVeg gab es 2017 fast 170 vegane Gastronomiebetriebe in großen und mittelgroßen Städten in Deutschland, das ist ein Anstieg der veganen Gastronomiebetriebe um rund $5 \% \mathrm{im}$ Vergleich zum Vorjahr (Statista 2017; ProVeg International 2019). Laut Food Report 2019 (Rützler und Reiter 2018) zeigt sich ein Trend, vermehrt Plant-based Food (pflanzenbasierte Lebensmittel, also Hülsenfrüchte, Getreide, Pilze, Algen etc.) zur Herstellung von Fleisch- und Fischersatzprodukten einzusetzen. Dabei haben Gäste ein wachsendes Interesse an Lebensmittelsicherheit und Lebensmittelqualität, welches Gastronomiebetriebe mit verstärkten Angaben zur Herkunft, Qualität und Zutaten der Gerichte befriedigen können (Rützler und Reiter 2018).

\subsection{Relevante Entwicklungen in den wichtigsten Herkunftsländern}

Sowohl in Österreich wie auch in den wichtigsten Herkunftsmärkten rücken verschiedene Ernährungsaspekte immer mehr in den Fokus von Diskussionen in Politik, Wissenschaft und Gesellschaft. Neben den Treibhausgasemissionen bei der Erzeugung von Lebensmitteln werden Fragen zu den Auswirkungen von Lebensmitteln auf die Gesundheit sowie zur „Ernährungskultur“ und zu kulinarischen Traditionen immer wichtiger. Der Genuss von regionalen kulinarischen Besonderheiten stellt für viele Urlauber einen wichtigen Teil des Reiserlebnisses dar. Der Konsum von biologischen regionalen Lebensmitten steigert demnach nicht nur die Gesundheit und das Wohlbefinden der Urlauber, sondern bietet auch die Möglichkeit, Traditionen und lokale Esskulturen in den Destinationen kennenzulernen. Regionale Lebensmittel sind beispielsweise für deutsche Urlauber sehr wichtig. Mehr als $60 \%$ ziehen lokale Speisen den von zu Hause gewohnten Speisen vor und knapp $72 \%$ finden „Essen und Trinken seien ein guter Weg, um andere Kulturen kennen zu lernen“ (LundDurlacher et al. 2016).

\subsection{Einfluss des Klimawandels bzw. Einfluss auf den Klimawandel}

\subsubsection{Einfluss des Klimawandels auf die Gastronomie}

Der Klimawandel kann sich auf die Gastronomie in unterschiedlicher Weise auswirken. Grundsätzlich kann der Klimawandel touristische Nachfrage verringern, mit entsprechenden Konsequenzen für die Gastronomie. Extremwetterereignisse wie kleinräumige Starkregen oder langfristig abnehmende Zeiträume mit Schneebedeckung sind Beispiele für Einnahmeausfälle. Zu den Risiken gehören aber auch Veränderungen der Kostenstruktur, zum Beispiel durch Ernteausfälle und die damit verbundene Notwendigkeit von Importen. In den letzten Jahren haben sich verschiedene Wetterphänomene negativ auf die landwirtschaftliche Produktion ausgewirkt. Laut Versicherungsverband Österreich ist die Anzahl der Naturkatastrophen in den letzten Jahren weltweit stark gestiegen. Auch Österreich ist gefährdet, vor allem durch Extremwetterereignisse wie kleinräumige Sturmböen, lokale Überflutungen, Schnee, Hagel und Hitzewellen (VVÖ 2016). Im Jahr 2017 gab es in der Schadenssaison 220 Schadensmeldungen pro Tag aufgrund von Hagelunwettern, Frost, Dürre und Überschwemmung, die einen Gesamtschaden von rund 250 Mio. $€$ in der Landwirtschaft, davon alleine 140 Mio. $€$ durch die Trockenheit insbesondere im Norden und Osten Österreichs verursachten (Österreichische Hagelversicherung 2017).

Bezüglich der Auswirkungen möglicher Steuererhöhungen bzw. ökonomischer Steuerungsmöglichkeiten wird auf Abschn. 4.3.2. und ergänzende Ausführungen in Kap. 13 verwiesen.

\subsubsection{Einfluss der Gastronomie auf den Klimawandel}

Die für die Erstellung des gastronomischen Angebotes wichtige Lebensmittelproduktion hat weitreichende Nachhaltigkeitsimplikationen, wie zum Beispiel Wasser- und Energieverbrauch, Landnutzung, Einsatz von Pestiziden, Artenvielfalt, den Einsatz genetisch modifizierter Organismen (GMOs), Tierschutz, Abfälle und Abwässer sowie Emis- 
sionen von Treibhausgasen (Vitousek et al. 1997; Zollitsch et al. 2007; Chapagain und Hoekstra 2008; Bhalli et al. 2009; Poore und Nemecek 2018). Insbesondere bezüglich des Klimawandels ist die Landwirtschaft in den vergangenen Jahren stärker in den Mittelpunkt der Emissionsminderungsdebatten gerückt. Global steht der Sektor für ungefähr $26 \%$ aller Emissionen, entsprechend 13,7 Mrd. Tonnen $\mathrm{CO}_{2}$ - $\mathrm{Äqui}-$ valenten (Poore und Nemecek 2018). Von diesen Gesamtemissionen der Produktionskette werden $61 \%$ durch die Landwirtschaft, d. h. die Produktion auf dem Bauernhof, verursacht. In Österreich ist die Landwirtschaft für 10,3 \% der Emissionen verantwortlich (Umweltbundesamt 2018). Treibhausgase entstehen aber auch bei der Produktion von Lebensmitteln, deren Verarbeitung, Verpackung, Transport, Kühlung und Lagerung, bei der Zubereitung der Speisen und durch Lebensmittelabfälle.

Verschiedene Studien haben gezeigt, dass Essgewohnheiten große Bedeutung für Emissionen haben und entsprechende Änderungen einen signifikanten Beitrag zur Emissionsminderung leisten könnten. Meier und Christen (2013) fanden beispielsweise, dass eine Änderung der Ernährungsgewohnheiten der Deutschen hin zu den Empfehlungen der Deutschen Ernährungskommission den Nahrungsmittelenergieverbrauch um $7 \%$ reduzieren könnten und Treibhausgasemissionen um $11 \%$, außerdem den Grund-/Oberflächenwasserverbrauch um $26 \%$ und die genutzte Landfläche um $15 \%$. Ähnliche Ergebnisse wurden für die USA von Tom et al. (2016) vorgelegt: Bei Umstellung auf einen kalorisch angepassten Nahrungsmittelkonsum könnten Energie-, Treibhausgas- und Grund-/Oberflächenwasserverbrauch um $9 \%$ gesenkt werden. Berechnungen für die EU gehen sogar davon aus, dass eine Halbierung des Fleisch-, Milchprodukt- und Eierverbrauchs Treibhausgasemissionen um bis zu $40 \%$ senken könnte und die Flächeninanspruchnahme um fast ein Viertel (Westhoek et al. 2014). Zu ähnlichen Ergebnissen kommen auch Poore und Nemecek (2018), die vorrechnen, dass eine globale Umstellung auf eine vegetarische Ernährung Emissionen an Treibhausgasen um 49 \% reduzieren könnte, entsprechend 5,5-7,4 Mrd. Tonnen $\mathrm{CO}_{2}$-Äquivalente. Auch das IPCC beziffert signifikante Einsparungsmöglichkeiten der Landwirtschaft von 7,2-11,0 Gt $\mathrm{CO}_{2}$-Äquivalente pro Jahr (IPCC 2014). Gegenwärtig gibt es jedoch wenig Anzeichen dafür, dass Essensgewohnheiten signifikant nachhaltiger werden (z. B. Gose et al. 2016 für Deutschland). In Österreich sind die Emissionen aus der Landwirtschaft seit 1990 zwar stark gesunken, jedoch 0,3 $\mathrm{Mt} \mathrm{CO}_{2}$-Äquivalente (3,8 \%) höher als im Klimaschutzgesetz vorgesehen. Auch stiegen die Emissionen in 2016 gegenüber dem Vorjahr um 1,5\% (Umweltbundesamt 2018).

\section{Klimaverträgliche Einkaufspolitik}

Der Einkauf von Lebensmitteln weist bereits eine hohe Klimarelevanz auf, da Energiekonsum und Treibhausgasemissio- nen stark von Produktionsweisen sowie Transportdistanzen der Lebensmittel abhängen. Die $\mathrm{CO}_{2}$-Äquivalenteemissionen pro transportierter Tonne Lebensmittel sind für Luftfracht am höchsten (rund $2 \mathrm{~kg}$ pro Tonne und Kilometer), gefolgt von Lkw $(0,13 \mathrm{~kg})$, Eisenbahn $(0,04 \mathrm{~kg})$ und Schifffahrt $(0,009 \mathrm{~kg}$ Hochseefrachtschiff, 0,034 kg Binnenfrachtschiff; Ministerium für Umwelt, Landwirtschaft, Ernährung, Weinbau und Forsten Rheinland-Pfalz 2015). Diese transportverursachten Emissionen fallen bei regionalen Lebensmitteln in geringerer Menge an. Ein weiterer klimarelevanter Aspekt sind die Produktionsweisen der Lebensmittel. So sind die $\mathrm{CO}_{2}-$ Emissionen von Gemüse in Treibhauskultur um das bis zu 50-Fache höher als von Gemüse in Freilandkultur (Jungbluth 2000). Auch Lebensmittel aus biologischer Erzeugung sind in der Regel klimaschonender, da sie auf energieintensiven und $\mathrm{CO}_{2}$-emittierenden Düngereinsatz verzichten und auf selbstregulierende Mechanismen zurückgreifen (Reganold und Wachter 2016).

\section{Energieverbrauch bei Lagerung und Zubereitung}

Bei der Lagerhaltung kommt es zum einen auf energieeffiziente Lager- und Kühlsysteme an, zum anderen aber auch auf eine optimierte Planung beim Lebensmitteleinkauf, damit nicht zu große Vorräte gehalten werden müssen und die Gefahr verdorbener Lebensmittel reduziert wird. Auch bei der Zubereitung geht es um die Energieeffizienz der Geräte, aber auch um effiziente Zubereitungsprozesse und darum, möglichst wenig Abfall zu produzieren.

Laut ÖGUT liegen die Energiekosten in der Gastronomie bei $5-6 \%$ des Umsatzes, entsprechend $100-135 \mathrm{kWh}$ pro $\mathrm{m}^{2}$ Betriebsfläche und Jahr bzw. $5-10 \mathrm{kWh}$ pro Mahlzeit. Der größte Energieverbrauch in der Gastronomie liegt in der Raumheizung, gefolgt von der Prozesswärme für die Küche, vor allem für das Garen von Speisen, aber auch für das Warmhalten und Erwärmen von Speisen, die Geschirrvorwärmung und die Geschirrreinigung. Kühl- und Gefriereinrichtungen nehmen an Bedeutung zu, da wegen der Flexibilität des Angebots immer mehr Tiefkühlprodukte verwendet werden (Bayer et al. 2011).

Daneben spielt die Art der eingesetzten Lebensmittel eine gewichtige Rolle. Neben den bereits erwähnten klimarelevanten Faktoren bei der Auswahl der Lebensmittel (kurze Transportwege, saisonale und biologische Lebensmittel) spielt auch Fleisch, v. a. Rindfleisch, eine große Rolle. Der Konsum von einem kg regional produziertem Rindfleisch entspricht Emissionen von 13-29 kg CO 2 -Äquivalenten (Gössling et al. 2011). Zur besseren Visualisierung: Eine Steakmahlzeit $(200 \mathrm{~g})$ entspricht demnach ungefähr $20-50 \mathrm{~km}$ Fahrt mit einem Pkw (im Durchschnitt $12 \mathrm{~kg} \mathrm{CO}_{2} / 100 \mathrm{~km}$; Schodl 2017).

Wie in allen anderen Prozessstufen im Gastronomiebetrieb ist auch bei der Zubereitung die Vermeidung von Lebensmittelabfall ein zu berücksichtigender Aspekt. Vermeidung 
von Lebensmittelabfällen in der Zubereitung beginnt mit der Planung der Speisekarte. Hier ist darauf zu achten, ob z. B. Reste, die bei der Zubereitung von bestimmten Gerichten anfallen, für andere verwendet werden können (z. B. Gemüsereste oder Knochen für Suppen). Auch sogenannte From-nose-to-tail-Konzepte können helfen vor allem tierische Lebensmittelabfälle zu vermeiden. Dabei wird bewusst jedes Teil des Tieres (,von Kopf bis Schwanz“) verwertet teilweise mit Rückgriff auf alte Rezepte. Allerdings ist hier eine gewisse Kreativität nötig, um z. B. Innereien in einer für den Kunden entsprechend attraktiven Form zuzubereiten und anzubieten. Auch die Rezeptur der Gerichte bietet einen wichtigen Angriffspunkt zur Abfallvermeidung. Je einfacher die Rezeptur der Gerichte, desto weniger Zubereitungsprozesse sind notwendig, wobei auch weniger Abfall entsteht, weil weniger unterschiedliche Zutaten verwendet werden müssen.

\section{Abfallaufkommen und -management}

Innerhalb der EU-28 werden pro Jahr rund 88 Mio. Tonnen an Lebensmittelabfall entlang der gesamten Versorgungskette entsorgt (Stenmarck et al. 2016). Lebensmittelabfall aus der Gastronomie wurde mit 11 Mio. Tonnen als eine der Hauptquellen identifiziert (Stenmarck et al. 2016). Scherhaufer et al. (2018) analysierten die mit diesen Lebensmittelabfällen zusammenhängenden Umweltauswirkungen. Die Ergebnisse zeigen, dass $186 \mathrm{Mt} \mathrm{CO}_{2}$-Äquivalente allein auf Lebensmittelabfälle in Europa zugeführt werden können.

Da die Produktion von Lebensmitteln energieintensiv ist, haben die Vermeidung und das Management von Lebensmittelabfällen große Bedeutung. Abfallmanagement bezieht sich auf alle Maßnahmen, die zur Vermeidung, Wieder- bzw. Weiterverwendung, Verwertung sowie geordneten Entsorgung von Abfällen eingesetzt werden. In der Gastronomie werden erhebliche Mengen von Abfällen erzeugt. In Deutschland rechnet etwa die DEHOGA (2016) in Gaststätten mit einem Volumen von 1,7 1 pro Gedeck. Deutlich höher sind die Abfallvolumen in Hotelbetrieben mit Gastronomie: In Betrieben mit 0 bis 2 Sternen geht die DEHOGA (2016) von 9,1 1 Abfall pro Übernachtung aus. Als Grund für die hohen Abfallmengen sieht die DEHOGA (2016) unter anderem die Verwendung vieler Einwegverpackungen, etwa beim Frühstück.

Grundsätzlich lässt sich zwischen vermeidbaren und unvermeidbaren Lebensmittelabfällen unterscheiden. Vermeidbare Abfälle sind solche, die zum Zeitpunkt des Wegwerfens noch für den menschlichen Konsum geeignet waren. Unvermeidbare Abfälle sind Lebensmittelbestandteile, die üblicherweise nicht gegessen werden (z. B. Knochen oder Schalen). Die Schweizer Organisation Confédération Suisse (2014) geht zum Beispiel davon aus, dass $70 \%$ aller Lebensmittelabfälle in Schweizer Restaurants vermeidbar sind. Eine detaillierte Studie der gesamten Versorgungskette zweier Schweizer Betriebe stellte fest, dass 7,7-10,7 \% der eingekauften Lebensmittel weggeworfen wurden, vor allem weil die servierten Portionen nicht aufgegessen wurden (Betz et al. 2015). Zwischen 78-92 \% der Abfälle waren damit vermeidbar. Dies hat auch ökonomische Implikationen, da der Wert der weggeworfenen Lebensmittel (10,5 t bzw. 16,5 t pro Jahr und Restaurant) von Betz et al. (2015) pro Betrieb auf rund $85.000 \mathrm{CHF}$ beziffert wurde.

In Österreich betragen laut einer Studie aus den Jahren 2015 bis 2019 (Hrad et al. 2016; Obersteiner et al. 2019) die vermeidbaren Speiseabfälle (ohne Zubereitungsreste) in der Außer-Haus-Verpflegung $3 \%$ (Restaurant) bis $60 \%$ (Eventcatering) des ausgegebenen Essens, wobei je nach Betriebstyp durchschnittlich 14-22\% der Lebensmittel ungenützt entsorgt werden. Cateringunternehmen und Großküchen im Gesundheitswesen zeigten aufgrund strengerer Hygienevorschriften mit $34 \%$ bzw. $28 \%$ signifikant höhere Verlustraten als Hotels (20\%) und reine Gastronomiebetriebe $(14 \%)$. Hochrechnungen ergaben, dass in Österreich jährlich rund 45.000 Tonnen vermeidbare Lebensmittelabfälle in der Gastronomie, 50.000 Tonnen in der Beherbergung, 61.000 Tonnen in der Gemeinschaftsverpflegung sowie 19.000 Tonnen in sonstigen Betrieben, wie z. B. Kaffeehäusern, anfallen (Unsicherheitsgrad von plus/minus $10 \%$ ). Unter Berücksichtigung von durchschnittlichen Einkaufspreisen landen damit in der heimischen Außer-Haus-Verpflegung pro Jahr Lebensmittel im Warenwert von rund 320 Mio. $€$ in der Mülltonne - das sind $8000 €$ pro Betrieb. Jährlich werden demnach rund 400.000 Tonnen $\mathrm{CO}_{2}$ durch die Produktion von ungenützten Lebensmitteln und deren anschließende Entsorgung als Abfall in der österreichischen Außer-Haus-Verpflegung verursacht (United Against Waste 2016).

\subsection{Anpassungs-, Minderungsmaßnahmen und Strategien}

\subsubsection{Handlungsfeld Einkauf}

Köche haben große Spielräume, um die Emissionsintensität des kulinarischen Angebots zu beeinflussen. Dies liegt an den sehr unterschiedlichen Emissionsintensitäten der Rohstoffe, die verwendet werden. Abb. 5.2 zeigt dies für die besonders wichtige Kategorie Fleisch. Die hier genannten Studien zeigen sehr deutlich, dass pro kg Huhn oder Schweinefleisch nur ein Bruchteil der Emissionen entsteht, die die Produktion von Rindfleisch verursacht (Poore und Nemecek 2018). Eine im Jahr 2012 veröffentlichte Studie zur Klimabilanz von Lebensmitteln zeigt auf, dass ein $\mathrm{kg}$ aus Brasilien importiertes Rindfleisch $335 \mathrm{~kg} \mathrm{CO}_{2}$-Äquivalente erzeugt, während Rindfleisch aus den Niederlanden nur auf $16 \mathrm{~kg} \mathrm{CO}_{2}$-Äquivalente kommt (Schmidinger und Stehfest 2012). Grund für diesen großen Unterschied ist die Einbeziehung des Flächenverbrauchs 
Abb. 5.2 $\mathrm{CO}_{2}$-Emissionen von Lebensmitteln bis $90 \mathrm{~kg} \mathrm{CO}_{2}^{-}$ Emissionen (Äquivalente) pro kg Erzeugnis - Variation basierend auf 570 Studien. (Datenquelle: Poore und Nemecek 2018; Grafik: Hannes Antonschmidt)

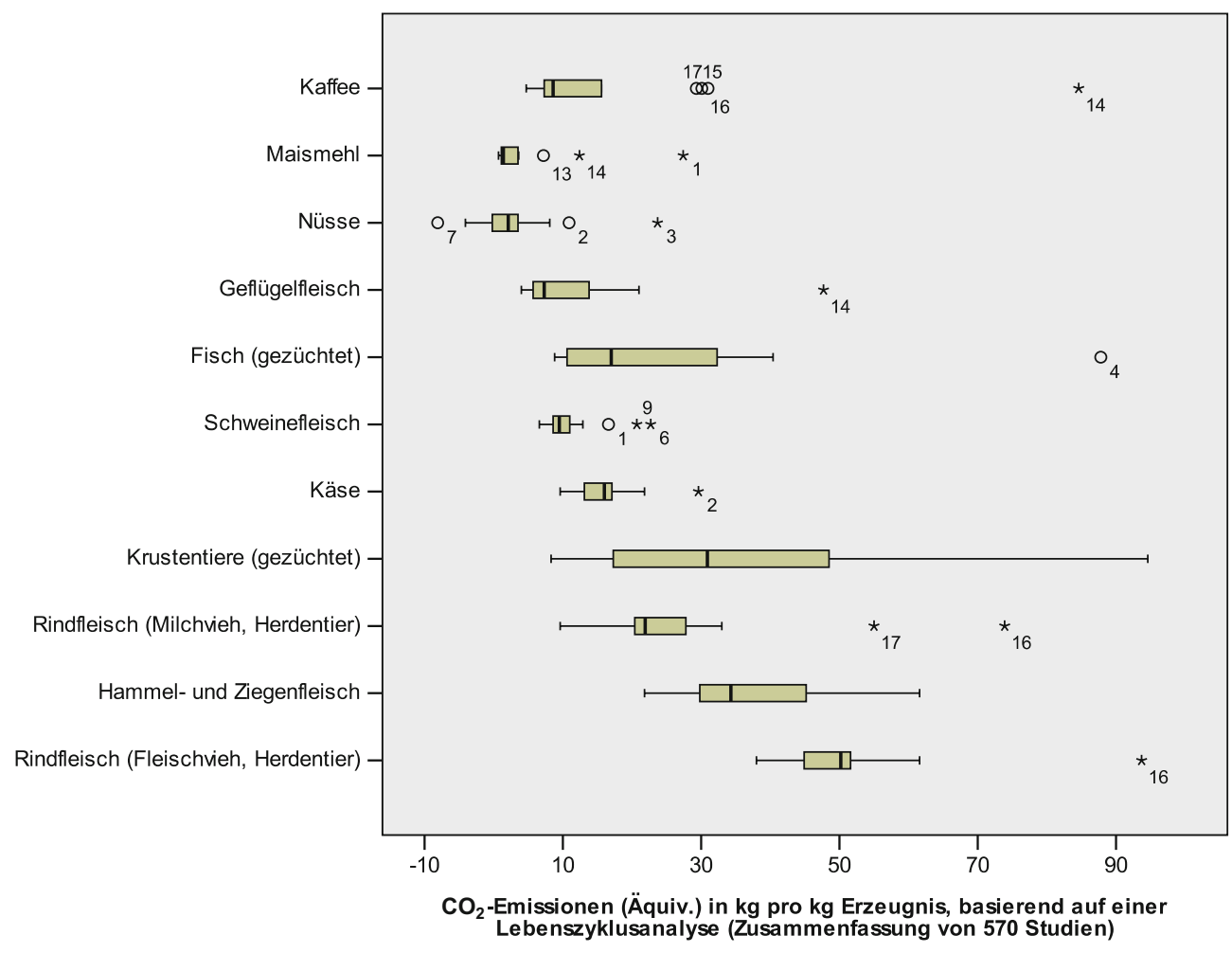

für die Rindfleischproduktion in das Berechnungsmodell (Life-Cycle-Assessment-Methode, LCA) und das damit verbundene $\mathrm{CO}_{2}$-Speicherpotenzial. Landwirtschaftlich genutzte Flächen binden weniger $\mathrm{CO}_{2}$ aus der Atmosphäre als Flächen mit natürlicher Vegetation (,missed potential carbon sink“). Da das $\mathrm{CO}_{2}$-Speicherpotenzial im brasilianischen Regenwald größer ist, fallen hier abgeholzte Regenwälder stärker ins Gewicht. Eine generelle Aussage, dass lokal produziertes Rindfleisch klimaschonender ist als importiertes, lässt sich daraus allerdings nicht ableiten, da die berechneten Treibhausgasemissionen vor allem von der Produktionsweise und -kette abhängen (intensive Rinderhaltung, importierte Futtermittel, Effizienz der Betriebe, Transporteffizienz etc.; Schmidinger und Stehfest 2012).

Durch das Angebot vegetarischer und veganer Menüalternativen können Emissionen erheblich reduziert werden.

Ein Metareview von Poore und Nemecek (2018) zeigt die während ihres Lebenszyklus von Lebensmitteln verursachten $\mathrm{CO}_{2}$-Emissionen nach Produktkategorien. Demnach zählen Fleischprodukte, Fisch und Meeresfrüchte zu den emissionsintensiven Produkten. Obst, Gemüse und Getreideprodukte verursachen hingegen geringe $\mathrm{CO}_{2}$-Emissionen. Gleichzeitig ergeben sich innerhalb der Kategorien z. T. erhebliche Spannbreiten, die v. a. auf Anbaumethode und Produktionsort zurückzuführen sind (Abb. 5.2 und 5.3). Der Einkauf aller Lebensmittel, die mit dem Flugzeug transportiert (z. B. Riesengarnelen, Viktoriabarsch) oder in geheizten Gewächshäusern (mit Ausnahme von Gewächshäusern, die mit nichtfossilen und $\mathrm{CO}_{2}$-neutralen Brennstoffen oder alternativen Energien beheizt werden) produziert wurden, ist emissionsintensiv. Wo Fleischgerichte angeboten werden, ist aus Klimaperspektive Huhn effizienter als Schweinefleisch, was wiederum besser ist als Lammfleisch. Ganz unten auf der Liste von Fleischgerichten steht Rindfleisch (Poore und Nemecek 2018). Auch Reis weist als Beilage hohe Emissionswerte auf. Für die Klimabilanz positiv ist dagegen die Nutzung von saisonalen Gemüsen, Kartoffeln und Getreide (Gössling et al. 2011). Eine größere Transparenz hinsichtlich der Energieintensität unterschiedlicher Nahrungsmittel wäre grundsätzlich wünschenswert.

Beim Einkauf von Restaurants schlägt sich auch das verwendete Verpackungsmaterial in der Klimabilanz nieder, wobei vor allem die Nutzung von Aluminiumfolie, deren Herstellung große Energiemengen erfordert, problematisch ist (Gössling et al. 2011). Auch im Bereich der Getränke gibt es große Unterschiede in der Energieintensität. Poore und Nemecek (2018) heben beispielsweise hervor, dass pro Liter Bier nur $20 \mathrm{~g} \mathrm{CO}_{2}$-Äquivalente für die Produktion und den Transport anfallen, wenn Stahlfässer verwendet werden. Bei Nutzung wiederverwertbarer Flaschen steigt die Treibhausgasbelastung um das 15- bis 40-Fache, auf 300-750 $\mathrm{g} \mathrm{CO}_{2}$ Äquivalent pro Liter.

Neben saisonalen Lebensmitteln kann auch der Einkauf regionaler Produkte einen erheblichen Unterschied bei der Klimabilanz machen, sofern es einen kombinierten Vertriebsweg gibt, der individuelle Transporte unnötig macht. Weltweit werden $17 \%$ aller Nahrungsmittel über Grenzen hinweg transportiert, zum Teil über große Entfernungen (Poore und 
Abb. 5.3 $\mathrm{CO}_{2}$-Emissionen von Lebensmitteln bis $11 \mathrm{~kg}$ $\mathrm{CO}_{2}$-Emissionen (Äquivalente) pro kg Erzeugnis - Variation basierend auf 570 Studien. (Datenquelle: Poore und Nemecek 2018; Grafik: Hannes Antonschmidt)

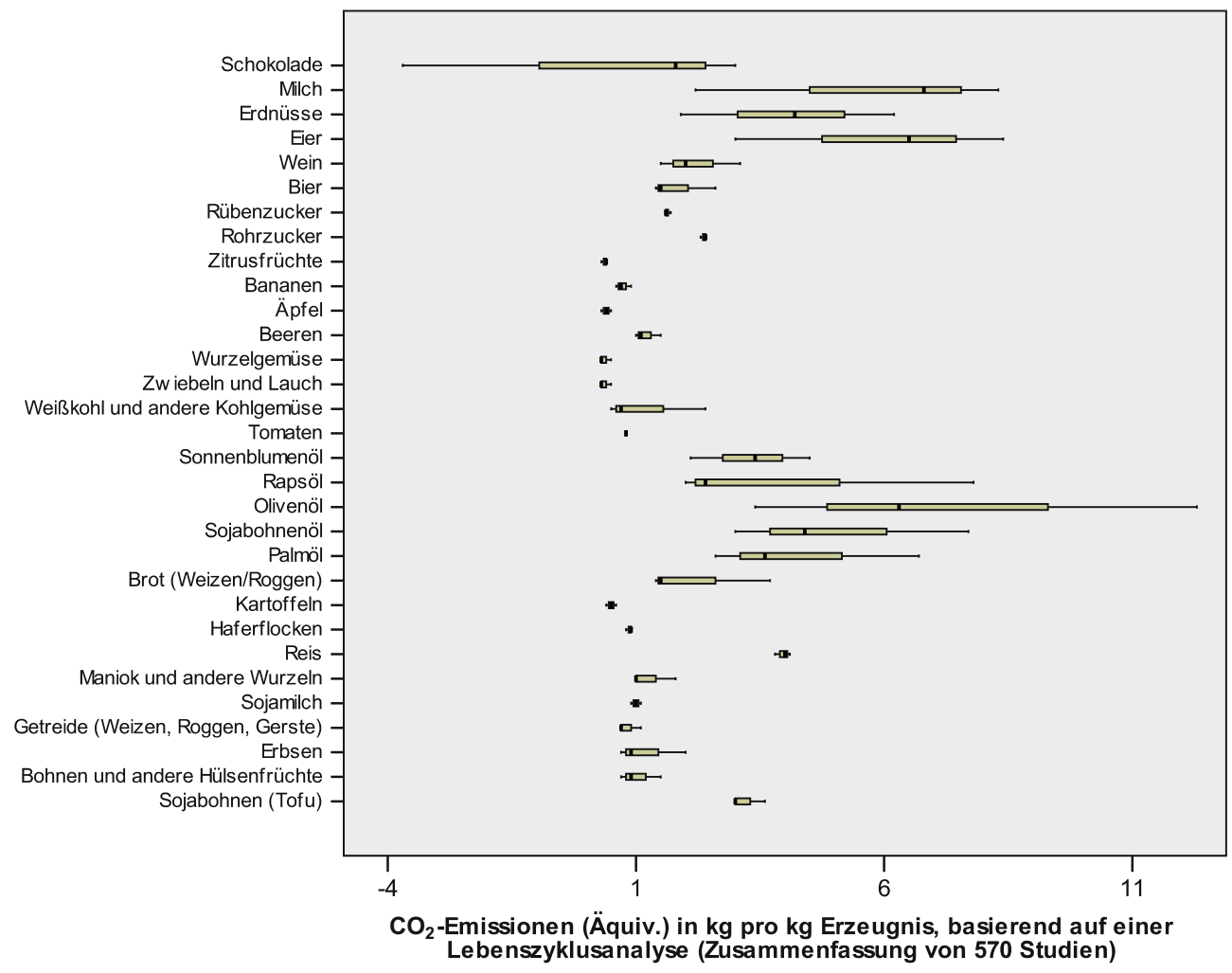

Nemecek 2018). Ein erheblicher Teil dieser Importe könnte vermieden werden. Es gibt unterschiedliche Definitionen für regionale Lebensmittel, die Distanzen von $100 \mathrm{~km}$ (Viabono in Deutschland) bis 400 Meilen (Green Restaurant Association, USA) als regional bezeichnen. In Österreich macht die Marke „Genuss Region Österreich“ regionale landwirtschaftliche Produkte sichtbar und stärkt das Verständnis für landwirtschaftliche Produktionsweisen und die Verbindung zwischen Naturlandschaft und Landwirtschaft. ${ }^{1}$ Auch das 2019 ins Leben gerufene Netzwerk Kulinarik hat das Ziel, regionale Landwirtschaftsprodukte zu stärken und als Plattform zur Vernetzung von Kulinarikinitiativen zu dienen. ${ }^{2}$ Zahlreiche Veröffentlichungen zeigen, dass regionale Lebensmittel bei Konsumenten einen hohen Stellenwert haben und dementsprechende Auszeichnungen ein Qualitätsmerkmal sind (z. B. Feucht und Zander 2018).

Da auch Touristinnen und Touristen einen großen Wert auf lokale Lebensmittel legen, kommt dem Aufbau lokaler Lieferantennetzwerke eine hohe Bedeutung zu (Hall und Gössling 2016). Mehr und mehr Hotels und Gastronomiebetriebe legen auch eigene Küchengärten an, die nicht nur Transportkilometer und Verpackungsmaterial vermeiden, sondern auch eine Attraktion für die Gäste darstellen. Auch der damit verbundene Einsatz saisonaler, biologischer Lebensmittel verringert $\mathrm{CO}_{2}$-Emissionen.

\footnotetext{
$1 \mathrm{http}: / /$ www.genuss-region.at.

${ }^{2} \mathrm{https} / / / \mathrm{b} 2 \mathrm{~b}$.amainfo.at/kulinarik/netzwerk-kulinarik-aktuelles/.
}

\subsubsection{Handlungsfeld Zubereitung der Speisen}

Die Zubereitung von Speisen betrifft vor allem Ressourceneffizienz bei der Zubereitung, die durch einen niedrigen Energieverbrauch, durch geringe Verschwendung (z. B. wenig Verschnitt) oder an den Grundsätzen der Suffizienz orientierte Zubereitungsarten (z. B. genaue Mengenplanung) erreicht wird (Lund-Durlacher et al. 2016).

Lebensmittelabfallmengen können reduziert werden, indem die Auswahl an Gerichten insgesamt eingeschränkt wird. Auch die Auswahl der Rezepturen bietet Möglichkeiten, Abfälle bei Lagerhaltung und Zubereitung zu reduzieren. Bei gleichbleibender Attraktivität der Speisen entsteht bei weniger Zutaten pro Gericht auch weniger Abfall (United Against Waste 2015).

Als wesentlicher Aspekt ist das Monitoring der vorhandenen Abfallmengen als Ausgangspunkt für zukünftige Einsparungen anzusehen. Es konnte in unterschiedlichsten Versuchen gezeigt werden, dass der Einsatz von Wiegesystemen in der Küche zu einer automatischen Verringerung der Abfallmengen führt (United Against Waste 2015).

Innovative, energieeffiziente Küchentechnik und moderne Zubereitungsmethoden wie Sous-Vide-Verfahren, Schockfrosten etc. bieten ein erhebliches Energieeinsparpotenzial. ${ }^{3}$ Für die Speisenzubereitung werden oft leistungsstarke Geräte eingesetzt, die $40 \%$ des Energieverbrauchs in der Küche

\footnotetext{
${ }^{3} \mathrm{https}: / /$ www.energie.ch/kochherd.
} 
verursachen und beim Startvorgang für Leistungsspitzen verantwortlich sind (WIFI 2002). Durch die Umstellung auf Induktionstechnologie kann viel Strom eingespart werden. Weitere Einsparungen sind durch gezielte Schulung des Personals (z. b. Motivation und Aufklärung), gezielten Geräteeinsatz, möglichst wenig Stand-by-Betrieb und den Einsatz von energieeffizienten Geräten möglich (WIFI 2002). Geschirrspüler und Kühlanlagen haben ebenfalls einen hohen Energiebedarf. Geschirrspüler an eine Warmwasserleitung anzuschließen sowie regelmäßige Wartung der Kühlanlagen, Ausnutzen der Volumina sowie richtige Temperatureinstellung sind weitere Einsparmaßnahmen. Durch Abwärmenutzung von Kühlanlagen und Küchengeräten zur Warmwassererwärmung sind weitere Energieeinsparungen realisierbar. Einsparmöglichkeiten bieten auch Lüftungsanlagen in Küche und Gasträumen durch optimale Dimensionierung bzw. effizienten Einsatz (richtige Temperatur- und Luftmengenwahl, regelmäßige Wartung und Verwendung eines Wärmetauschers; WIFI 2002).

Wie die DEHOGA (2016) vorrechnet, haben viele Maßnahmen Amortisierungszeiträume, die bei nur wenigen Monaten liegen. Andere Maßnahmen, wie die Einstellung idealer Kühltemperaturen, die nächtliche Abschaltung von Eismaschinen oder die korrekte Standortwahl für Kühl- und Gefriergeräte, sind sogar kostenfrei. Mindestens 30\% der Energie können in der Gastronomie durchschnittlich eingespart werden, sofern alle Maßnahmen mit Amortisierungszeiträumen von bis zu drei Jahren durchgeführt werden (DEHOGA 2016). Bei Einzelmaßnahmen, wie der Anschaffung effizienter Kühlgeräte, rechnet die Austrian Energy Agency (2018) sogar mit Einsparungen von bis zu $50 \%{ }^{4}$

\subsubsection{Handlungsfeld Präsentation von Speisen}

In der Gastronomie können viele Strategien helfen, bestimmte Gerichte stärker zu vermarkten und Abfälle zu vermeiden. Restaurants können beispielsweise ihren Gästen klimaschonende Gerichte ans Herz legen, indem sie diese als Tagesgerichte hervorheben oder durch das Personal empfehlen lassen. Empfehlungen können sich dabei an allgemeinen Qualitätsmerkmalen orientieren, also der Verwendung lokaler Zutaten oder geschmacklichen Eigenschaften. Eine Voraussetzung für solche Strategien ist es, Servicepersonal auch hinsichtlich der Umwelteigenschaften verschiedener Lebensmittel einzuweisen. Gerichte mit einem hohen Anteil lokaler oder nachhaltig produzierter Lebensmittel können auch mit einem Qualitätssiegel in der Karte hervorgeho-

\footnotetext{
${ }^{4}$ Informationen zu besonders effizienten Geräten finden sich z. B auf der Seite www.b2b.topprodukte.at. Im Jahr 2018 konnten Gastronomie- und Beherbergungsbetriebe auch am Klimaschutzwettbewerb "ClimaHost" teilnehmen (www.climahost.eu).
}

ben werden. Eine Studie von Visschers und Siegrist (2015) zeigte etwa, dass ein „Klimafreundliche-Wahl“-Hinweis in der Speisekarte die Wahl solcher Gerichte um $10 \%$ erhöhte (Abschn. 5.4.5).

In der Gastronomie werden Lebensmittel insbesondere dort verschwendet, wo Büffets angeboten werden. Es empfiehlt sich daher, Büffets nach bestimmten Aspekten auszurichten. Dazu gehört, Gästen kleinere Teller anzubieten, um einer Überladung und damit Tellerresten vorzubeugen. Die Verwendung von kleineren und flacheren Gebinden am Büffet erlaubt eine bedarfsorientierte Wiederauffüllung mit weniger Lebensmitteln bei permanentem umfassenden Angebot und gleichzeitiger Vermeidung von Abfällen. Zu Lebensmittelabfallvermeidungsmaßnahmen im Büffetbereich zählen auch die klare Kennzeichnung der Speisen und der Verzicht auf essbare Dekoration. Wo möglich kann durch Frontcooking ebenfalls besser auf den tatsächlichen Bedarf reagiert und eine Überproduktion an Speisen vermieden werden. Portionsgrößen wählbar zu machen bzw. Gästen anzubieten, nichtkonsumiertes Essen mit nach Hause zu nehmen, sind ebenfalls effektive Maßnahmen zur Vermeidung von Tellerresten. Dazu können beispielsweise Behälter aus Papier oder Kunststoff verwendet werden (nicht aus Aluminium, das sehr energieintensiv produziert werden muss), wobei allgemeingültige Hygienevorschriften zu berücksichtigen sind (Gössling 2010; Martin-Rios et al. 2018). ${ }^{5}$ Sofern relevant, ist eine Bezahlung nach Gewicht statt nach Portionsgröße ebenfalls eine Möglichkeit, das Aufkommen an Tellerabfällen bereits am Büffet zu reduzieren. Ein weiterer klimarelevanter Aspekt ist vegetarische Alternativen in besserer Erreichbarkeit anzubieten und so den Fleischkonsum zu reduzieren.

\subsubsection{Handlungsfeld Abfallmanagement}

Die „Hierarchie der Lebensmittelabfälle“ (Papargyropoulou et al. 2014) zeigt Möglichkeiten des Abfallmanagements durch Vermeidung, Wiederverwendung, Recycling und andere Rückgewinnungsmaßnahmen auf. Die Entsorgung bleibt dabei der letzte Ausweg. Die beste Lösung besteht darin, Lebensmittelabfälle gar nicht erst entstehen zu lassen. Eine wichtige Maßnahme zur Identifizierung von Reduktionspotenzialen ist das Messen der Abfallmengen. Die Initiative United Against Waste ${ }^{6}$ stellt beispielsweise Onlineinstrumente zur Erfassung der Lebensmittelabfälle bereit. Durch die Erfassung lässt sich feststellen, wie viel, welche Art und wo genau Abfall anfällt. Mit diesen Informationen können Ursachen analysiert und Abfälle vermieden werden. Uni-

\footnotetext{
${ }^{5}$ Eine erfolgreiche Initiative in Österreich zur Vermeidung von Lebensmittelabfällen am Büffet ist die Tafelbox (https://tafelbox.at/).

${ }^{6} \mathrm{http}: / /$ united-against-waste.at/erheben/abf/.
} 
ted Against Waste Österreich präsentiert auch zahlreiche Lösungsansätze zur Vermeidung von Lebensmittelabfällen in der Broschüre „Lebensmittelabfälle vermeiden, Kosten sparen \& Umwelt schützen“ (United Against Waste 2018). Dazu gehören Maßnahmen wie die Optimierung der Speisenangebotsplanung (basierend auf Reservierungsvorhersagen, demografischen Charakteristika und Speisepräferenzen der Gäste), Vermeidung des Verderbs der Lebensmittel sowie Vermeidung von Abfall am Büffet und auf den Gästetellern. Sehr detailliert nennen Betz et al. (2015) weitere Maßnahmen zur Reduzierung des Lebensmittelabfalls, die sich auf den Einkauf, die Lagerung, Zubereitung, Präsentation und Beeinflussung des Gästeverhaltens beziehen. Neben den Lebensmittelabfällen fallen in der Gastronomie auch große Mengen von Verpackungsmüll an, die durch gezielte Maßnahmen wie Einkauf in Großmengen, wiederverwendbare Verpackungen, Spendersysteme, Sammel- und Recyclingsysteme etc. reduziert werden können.

$\mathrm{Zu}$ viel produzierte Lebensmittel können, bevor sie Abfall werden, an karitative Einrichtungen, „Foodsharing“-Organisationen oder andere Institutionen gespendet werden. „Late night offers" über entsprechende Onlineplattformen sind ein weiterer Weg, Lebensmittel ihrem tatsächlichen Zweck zuzuführen. Im Fall der Tellerreste ist die aktive Anpreisung von Mitnahmeboxen (vgl. Tafelbox) zu empfehlen. Für all diese Beispiele ist vor allem die indirekte Einsparung an Treibhausgasen relevant. Durch die Weitergabe der Speisen müssen diese vom Endkonsumenten nicht zusätzlich gekauft werden und können auf lange Sicht dementsprechend weniger produziert werden. Das heißt, es kommt zu verringerten Emissionen durch die Lebensmittelproduktion.

Für nicht vermeidbare Lebensmittelabfälle wie Zubereitungsreste und für dennoch angefallene vermeidbare Abfälle ist es wesentlich, sie einer entsprechenden Entsorgung zuzuführen. Eine getrennte Sammlung der Bioabfälle und nachfolgende Verwertung in Kompostier- oder Biogasanlagen hilft zusätzlich Klimaemissionen einzusparen. Wenn entsprechende Systeme nicht seitens kommunaler oder privater Entsorger angeboten werden, kann hier mittlerweile auch auf Angebote zur z. B. Selbstkompostierung zurückgegriffen werden. Auch eine energetische Nutzung der Abfälle ist möglich.

\subsubsection{Handlungsfeld Kommunikation}

Um klimarelevante Prozesse umzusetzen, sind zum einen die Unterstützung und das Engagement der Führungskräfte notwendig, zum anderen aber auch Bewusstseinsbildung und Schulung der Mitarbeiter sowie Gästekommunikation. Gerade Urlaubsgäste sind sehr daran interessiert, über die Herkunft, Produktionsmethoden und Nährwerte ihrer Speisen informiert zu werden (Lund-Durlacher et al. 2016; Juvan et al.
2018) und diese Informationen können Verhaltensänderungen bewirken. Experimentelle Studien mit unterschiedlichen Informationsinstrumenten bei der Speisenausgabe zeigen, dass eine Verhaltenssteuerung des Gästeverhaltens durch adäquate Kommunikation möglich ist. So konnten durch den Einsatz von Beschilderungen am Büffet und im Gastraum Tellerreste um durchschnittlich knapp $15 \%$ reduziert und der Konsum lokaler Produkte um durchschnittlich rund $130 \%$ gesteigert werden (Antonschmidt und Lund-Durlacher 2018). Damit bieten die Kennzeichnung und transparente Information zu klimarelevanten Aspekten der Speisen eine gute Möglichkeit, das Gästeverhalten positiv zu beeinflussen. Wie im Abschn. 5.4.3 hervorgehoben, können auch Kennzeichnungen von Speisen als biologisch, lokal oder klimaschonend zu signifikanten Änderungen in der Menüwahl führen. Der Effekt solcher Label scheint dabei mit der Zeit zuzunehmen (Visschers und Siegrist 2015), vermutlich aufgrund des Wiedererkennungswertes und der Wahrnehmung als Qualitätskennzeichen.

Eine Reihe von Studien widmet sich dem Thema „Nudging“. Bei dieser Technik wird der Konsument durch geschickte Manipulation in seinem Konsumverhalten beeinflusst, ohne dass er diese Beeinflussung bewusst wahrnimmt und ohne dass seine Wahlfreiheit z. B. durch Verbote eingeschränkt wird. Im Lebensmittelbereich unterscheiden Lehner et al. (2016) vier Erfolg versprechende Nudging-Strategien: Verfügbarmachung einfacher Informationsmittel (Hervorheben bestimmter für die Konsumentscheidung relevanter Informationen, z. B. Gesundheitswirkungen, und Einsatz von Referenzwerten für Konsumverhalten, z. B. Einsatz roter Kartoffelchips in bestimmten Intervallen einer Verpackungsrolle), Veränderung von Verfügbarkeit und Sichtbarkeit der Lebensmittel, Beeinflussung der Portionsgröße sowie soziale Normen und Idealverhalten, wobei das Konsumverhalten auch durch die Präsenz anderer Konsumenten beeinflusst wird. Zudem kann durch das direkte Aufzeigen ,richtigen“ Verhaltens (z. B. durch Schilder) das Verhalten von Konsumenten in eine Richtung gelenkt werden.

Verschiedene Studien belegen die Wirksamkeit der genannten Nudging-Instrumente. Wansink und van Ittersum (2003) zeigen, dass eine Veränderung der Glasform den Getränkekonsum beeinflusst, wobei höhere Gläser zu niedrigerem Konsum führen (bei gegensätzlicher Wahrnehmung seitens der Gäste). Freedman und Brochado (2010) verändern die Portionsgrößen in einem All-you-can-eat-Restaurant und zeigen, dass durch die Verringerung der Portionsgrößen Verzehr- und Abfallmengen sinken. Kallbekken und Saelen (2013) variieren die Tellergrößen und setzen zudem Hinweisschilder zum „richtigen“ Verhalten am Büffet ein, wodurch eine Reduktion der Lebensmittelabfälle um $20 \%$ erreicht wird. 


\subsection{Handlungsoptionen, Kommunikations- und Forschungsbedarf}

\subsubsection{Ansatzpunkte für Maßnahmen, die Überwindung von Barrieren und Kooperation}

Zentrale Ansatzpunkte für Maßnahmen in der Gastronomie sind die Gestaltung eines klimaschonenden Speisenangebots und damit verbunden einer klimaschonenden Einkaufspolitik, Energieeffizienzmaßnahmen sowie die Einbindung der Mitarbeiterinnen und Mitarbeiter sowie der Gäste in diese Maßnahmen.

Kooperationen zwischen regionaler Landwirtschaft, Großhandel und Gastronomiebetrieben befördern eine klimaschonende Einkaufspolitik. Der Aufbau von Netzwerken ermöglicht den Austausch von Informationen bezüglich Lebensmittelbedarf der Gastronomiebetriebe und Lebensmittelangebot der Landwirtschaftsbetriebe, ermöglicht den Aufbau regionaler Distributionsnetzwerke und kann somit regionale, biologisch angebaute Lebensmittel leichter verfügbar machen. ${ }^{7}$

Zur Reduktion von Treibhausgasemissionen können weitere Förderprogramme zur Unterstützung der Implementierung klimarelevanter Maßnahmen in der Gastronomie (Förderung innovativer Küchentechnologie, Abfallmanagement etc.) initiiert werden, die mit Angeboten einer kostenfreien Klimaberatung, in der Energiesparaspekte, klimaschonende Gerichte, Abfallmanagement und Kundenkommunikation angesprochen werden, gekoppelt werden sollten.

\subsubsection{Ansatzpunkte für die Akzeptanz von Maßnahmen, Information und Bewusstseinsbildung}

Zur Umsetzung klimarelevanter Prozesse sind das Engagement der Führungskräfte, Bewusstseinsbildung und Schulung der Mitarbeiter sowie Gästekommunikation notwendig.

Eine breit angelegte nationale Energiesparkampagne wirkt bewusstseinsbildend und kann Unternehmerinnen und Unternehmer sowie deren Personal zu energieeffizientem Verhalten bewegen. Durch den Aufbau von Wissensnetzwerken (Abschn. 5.5.1 Kooperationen) und Mitarbeiterschulungen können klimaschonende Speisenangebote und Küchenprozesse implementiert werden.

Auch der Einbau von Nachhaltigkeitsaspekten, insbesondere des Klimawandels, in die Lehrpläne (Curricula) der einschlägigen Berufsschulen, höheren Bundeslehranstalten

\footnotetext{
${ }^{7}$ Nationale Initiativen in Österreich dazu sind das Netzwerk Kulinarik (https://b2b.amainfo.at/kulinarik/) sowie die Genussregionen Österreich (https://www.genussregionen.at/).
}

(HBLA) sowie Fachhochschul- und Universitätslehrgänge würde zu einer erhöhten Sensibilisierung und zu gezieltem Wissensaufbau führen.

\subsubsection{Wissenslücken und Forschungsbedarf}

Forschungsrelevante Fragestellungen beziehen sich insbesondere auf österreichspezifische Einsichten zum Energieverbrauch, der Lebensmittelnutzung, der Abfallgenerierung sowie Einstellungen von Köchen, F\&B-Managern, Eigentümern und Gästen. Besonders interessant sind Forschungsansätze, die eine potenzielle Verhaltenssteuerung von Unternehmen und Gästen untersuchen.

Im Detail beziehen sich forschungsrelevante Fragestellungen auf:

- detaillierte Analysen zu Energiekosten und Einsparpotenzialen in der Gastronomie,

- detaillierte Analysen zur Gestaltung und Akzeptanz klimaschonender Speisenangebote,

- Kooperationen unter den Betrieben: Governance-Prozesse zum Aufbau von Multi-Stakeholder-Netzwerken,

- Gastronomiebetriebe: Auf welche Weise können Unternehmen ,incentiviert“" werden, klimarelevante Investitionen zu tätigen?

- Die Gäste und das Gästeverhalten: Wie, ohne das Urlaubserlebnis zu schmälern, kann eine Verhaltensänderung hin zu einem klimaschonenden Speisenkonsum bewirkt werden?

\subsection{Zusammenfassung}

Die Gastronomie liefert wichtige Serviceleistungen an Touristen und ist gleichzeitig auch ein wichtiger Abnehmer der Landwirtschaft und der Nahrungsmittelindustrie. Für Österreich-Urlauber spielt das Thema Kulinarik und regionale Speisen eine große Rolle, wobei in den letzten Jahren auch der klimarelevante Trend zur Reduzierung des Fleischkonsums in den Hauptherkunftsmärkten festzustellen ist (hohe Übereinstimmung, mittlere Beweislage).

Klimabezogene Risiken für Gastronomiebetriebe bestehen vor allem in einer durch Ernteausfälle möglichen Einschränkung der Nahrungsmittelversorgung, aber auch im Anfallen höherer Kosten durch die Notwendigkeit von Importen sowie die zur Erreichung der Klimaziele notwendige zusätzliche Besteuerung von Energie bzw. $\mathrm{CO}_{2}$-intensiven Produkten (niedrige Übereinstimmung, schwache Beweislage).

Die Gastronomie hat insbesondere auch durch den Einsatz landwirtschaftlicher Produkte einen erheblichen Einfluss auf den Klimawandel. Neben den in der Lebensmittelproduktion in unterschiedlicher Intensität anfallenden Treibhausgas- 
emissionen entstehen auch bei der Lebensmittelverarbeitung, Transport, Kühlung, Lagerung, bei der Speisenzubereitung und durch Lebensmittelabfälle Treibhausgase (hohe Übereinstimmung, starke Beweislage).

Die Möglichkeiten, Treibhausgasemissionen zu reduzieren, sind für Gastronomiebetriebe groß. So führen Entscheidungen für ein klimaschonendes Speisenangebot (vegetarische und vegane Speisen) sowie eine klimaschonende Einkaufspolitik (verstärkte Nutzung von regionalen, biologisch und saisonal produzierten Lebensmitteln) zu erheblichen Einsparungen von Treibhausgasen (hohe Übereinstimmung, starke Beweislage). Bei der Zubereitung der Speisen trägt eine innovative und energieeffiziente Küchentechnik zur Senkung des Energieverbrauchs und zum Abbau der Energieleistungsspitzen bei, wobei die Umsetzung vieler Einsparmaßnahmen auch Kostensenkungen nach sich ziehen (mittlere Übereinstimmung, schwache Beweislage). Auch die Reduzierung bzw. Weiterverwertung von Lebensmittel- und Verpackungsabfällen durch ein effizientes F\&B-Management trägt positiv zur Klimabilanz der Gastronomiebetriebe bei (hohe Übereinstimmung, mittlere Beweislage).

Die Gäste sowie deren Konsumverhalten spielen bei der Transformation zu klimaschonenden Gastronomieangeboten eine wesentliche Rolle (mittlere Übereinstimmung, schwache Beweislage). Deshalb ist zum einen eine qualitative hochwertige Zusammenstellung von klimaschonenden Angeboten unumgänglich, zum anderen bieten die Präsentationsmöglichkeiten der Speisen (Nudging-Techniken) sowie eine transparente klimarelevante Information vor und während der Konsumation (z. B. Kennzeichnung des Treibhausgasfußabdrucks für Gerichte) eine wichtige Rolle (mittlere Übereinstimmung, schwache Beweislage).

Zusammenfassend ist festzustellen, dass neben vielen einzelnen Maßnahmen in den Bereichen Einkauf, Zubereitung, Präsentation und Abfallmanagement, die Bereiche Aus- und Weiterbildung der Mitarbeiter sowie Gästekommunikation äußerst wichtige flankierende Bereiche zur erfolgreichen Umsetzung von Klimaschutzmaßnahmen in Küche und im F\&B-Bereich sind (hohe Übereinstimmung, mittlere Beweislage). Auch die volle Unterstützung des Managements für die Einführung und Weiterentwicklung eines nachhaltigen Gastronomieangebots ist unabdingbar.

\section{Kernaussagen - Kapitel 5}

- Klimabezogene Risiken für Gastronomiebetriebe bestehen in einer durch den Klimawandel bedingten verringerten touristischen Nachfrage (z. B. durch Extremwetterereignisse, wie kleinräumige Starkregen oder langfristig abnehmende Zeiträume mit Schneebedeckung). Zu den Risiken gehören aber auch Veränderungen der Kostenstruktur, zum Beispiel durch Ernteausfälle und die damit verbundene Notwendigkeit von Importen (mittlere Übereinstimmung, schwache Beweislage).

- Die Gastronomie hat durch den Einsatz landwirtschaftlicher Produkte einen Einfluss auf den Klimawandel. Treibhausgase entstehen in der Lebensmittelproduktion, bei der Lebensmittelverarbeitung, Transport, Kühlung, Lagerung, bei der Speisenzubereitung und durch Lebensmittelabfälle (hohe Übereinstimmung, starke Beweislage).

- Möglichkeiten für Gastronomiebetriebe, Treibhausgasemissionen zu reduzieren, bieten u. a. der Einsatz innovativer und energieeffizienter Küchentechnik, die Gestaltung eines weniger treibhausgasintensiven Speisenangebots durch verstärkte Nutzung von regionalen, biologisch und saisonal produzierten Lebensmitteln, ein größeres Angebot vegetarischer bzw. veganer Speisen sowie die Reduzierung und Weiterverwertung von Lebensmittel- und Verpackungsabfällen (hohe Übereinstimmung, starke Beweislage).

- Die Gäste sowie deren Konsumverhalten spielen bei der Transformation zu klimaschonenden Gastronomieangeboten eine wesentliche Rolle (mittlere Übereinstimmung, schwache Beweislage). Präsentationsmöglichkeiten der Speisen (Nudging-Techniken) sowie eine transparente klimarelevante Information vor und während der Konsumation (z. B. Kennzeichnung des Treibhausgasfußabdrucks für Gerichte) spielen eine wichtige Rolle (mittlere Übereinstimmung, schwache Beweislage).

- Die Mitarbeiterinnen und Mitarbeiter spielen bei der Umsetzung der Maßnahmen eine wichtige Rolle und müssen entsprechend ausgebildet sein. Deshalb sind entsprechende Schulungen und Weiterbildung unumgänglich (hohe Übereinstimmung, mittlere Beweislage).

\section{Literatur}

Antonschmidt, H. \& Lund-Durlacher, D. (2018) Can direct communication at the point of consumption reduce the attitude-behavior gap regarding food waste in hotels? In: J. Pearce (Hrsg.) Conference Proceedings of BEST EN Think Tank XVIII: Innovation and Progress in Sustainable Tourism, S. 19-30. James Cook University, Townsville, Australien. Online unter: http://www.besteducationnetwork. org/?module=file\&act=procFileDownload\&file_srl=16042\&sid=3be 9d5e8fb9472514633cf2760319fdd\&module_srl=879.pdf (letzter Zugriff: 28.05.2020).

Austrian Energy Agency (2018) Erstes EU-Energielabel im gewerblichen Bereich. Österreichische Energieagentur - Austrian Energy Agency, Wien, Österreich. Online unter: https://www.energyagency. at/aktuelles-presse/news/detail/artikel/erstes-eu-energielabel-im-gewerblichen-bereich.html?no cache $=1 \& \mathrm{~L}=0 \& \mathrm{cHash}=6139 \mathrm{be} 94 \mathrm{a} 76 \mathrm{~b}$ 3064970ffc1326259fb3 (letzter Zugriff: 14.05.2019). 
Bayer, G., Sturm, T. \& Hinterseer, S. (2011) Kennzahlen zum Energieverbrauch in Dienstleistungsgebäuden. ÖGUT - Österreichische Gesellschaft für Umwelt und Technik, Wien, Österreich. Online unter: https://www.oegut.at/downloads/pdf/e_kennzahlen-ev-dlg_ zb.pdf?m=1314366493 (letzter Zugriff: 21.0.2020).

Betz, A., Buchli, J., Göbel, C. \& Müller, C. (2015) Food waste in the Swiss food service industry-Magnitude and potential for reduction. Waste Management 35(1), 218-226. DOI: https://doi.org/10.1016/j. wasman.2014.09.015

Bhalli, J. A., Ali, T., Asi, M.R., Khalid, Z.M., Ceppi, M. \& Khan, Q.M. (2009) DNA damage in Pakistani agricultural workers exposed to mixture of pesticides. Environmental and Molecular Mutagenesis 50(1), 37-45. DOI: https://doi.org/10.1002/em.20435

Chapagain, A.K. \& Hoekstra, A.Y. (2008) The global component of freshwater demand and supply: An assessment of virtual water flows between nations as a result of trade in agricultural and industrial products. Water International 33(1), 19-32. DOI: https://doi. org/10.1080/02508060801927812

Confédération Suisse (2014) Gaspillage alimentaire dans le commerce de détail et la restauration en Suisse. Schweizerische Eidgenossenschaft, Bern, Schweiz. Online unter: http://www.news.admin.ch/ NSBSubscriber/message/attachments/37372.pdf (letzter Zugriff: 03.06.2020)

DEHOGA (2016) Nachhaltiges Wirtschaften in Hotellerie und Gastronomie: Tipps und Handlungsempfehlungen. Deutscher Hotelund Gaststättenverband e. V. (DEHOGA Bundesverband), Berlin, Deutschland. Online unter: https://www.dehoga-bundesverband. de/fileadmin/Startseite/05_Themen/Energie/DEHOGA_Umweltbroschu_re_Oktober_2016.pdf (letzter Zugriff: 03.06.2020).

FAO, IFAD \& WFP (2013) The State of Food Insecurity in the World 2013: the multiple dimensions of food security. Food and Agriculture Organisation of the United Nations (FAO), International Fund for Agricultural Development (IFAD) und United Nations World Food Programme (WFP), Rom, Italien. Online unter: http://www.fao.org/ 3/a-i3434e.pdf (letzter Zugriff: 21.04.2020).

Feucht, Y. \& Zander, K. (2018) Consumers' preferences for carbon labels and the underlying reasoning. A mixed methods approach in 6 European countries. Journal of Cleaner Production 178, 740-748. DOI: https://doi.org/10.1016/j.jclepro.2017.12.236

Freedman, M.R. \& Brochado, C. (2010) Reducing portion size reduces food intake and plate waste. Obesity 18(9), 864-1866. DOI: https:// doi.org/10.1038/oby.2009.480

Fritz, O., Laimer, P., Ostertag-Sydler, J., \& Weiß, J. (2019) Bericht über die Bedeutung, Entwicklung und Struktur der österreichischen Tourismus- und Freizeitwirtschaft im Jahr 2018. Österreichisches Institut für Wirtschaftsforschung \& Statistik Austria (Hrsg.), Wien, Österreich. Online unter: https://www.wifo.ac.at/wwa/pubid/61799 (letzter Zugriff: 06.12.2019).

Gose, M., Krems, C., Heuer, T. \& Hoffmann, I. (2016) Trends in food consumption and nutrient intake in Germany between 2006 and 2012: results of the German National Nutrition Monitoring (NEMONIT). British Journal of Nutrition 115(8), 1498-1507. DOI: https:// doi.org/10.1017/S0007114516000544

Gössling, S. (2010) Carbon management in tourism. Routledge, London, Vereinigtes Königreich.

Gössling, S., Garrod, B., Aall, C., Hille, J. \& Peeters, P. (2011) Food management in tourism: reducing tourism's carbon 'foodprint'. Tourism Management 32(3), 534-543. DOI: https://doi.org/10.1016/j. tourman.2010.04.006

Hall, C.M. \& Gössling, S. (Hrsg.) (2016) Food tourism and regional development. Routledge, London, Vereinigtes Königreich.

Hrad, M., Ottner, R., Lebersorger, S., Schneider, F. \& Obersteiner, G. (2016) Vermeidung von Lebensmittelabfall in Gastronomie, Beherbergung und Großküchen - Erweiterung weitere Betriebe. Endbericht. Institut für Abfallwirtschaft, Universität für Bodenkultur Wien, Österreich. Online unter: https://united-against-waste.at/wp-content/
uploads/2015/05/Endbericht_BOKU_2016_02_19.pdf?fa6be0 (letzter Zugriff: 21.04.2020).

IPCC (2014) Summary for Policymakers. In: Edenhofer, O., Pichs-Madruga, R., Sokona, Y., Farahani, E., Kadner, S., Seyboth, K., Adler, A., Baum, I., Brunner, S., Eickemeier, P., Kriemann, B., Savolainen, J., Schlömer, S., von Stechow, C., Zwickel, T. \& Minx, J.C. (Hrsg.) Climate Change 2014: Mitigation of Climate Change. Contribution of Working Group III to the Fifth Assessment Report of the Intergovernmental Panel on Climate Change, S. 1-30. Cambridge University Press, New York, NY, USA. Online unter: https://www.ipcc.ch/ site/assets/uploads/2018/02/ipcc_wg3_ar5_summary-for-policymakers.pdf (letzter Zugriff: 21.04.2020).

Jungbluth, N. (2000) Umweltfolgen des Nahrungsmittelkonsums. ÖkoInstitut e. V., Freiburg, Deutschland. Online unter: https://www.oeko. de/oekodoc/80/2000-012-de.pdf (letzter Zugriff: 21.04.2020).

Juvan, E., Grün, B. \& Dolnicar, S. (2018) Biting off more than they can chew: food waste at hotel breakfast buffets. Journal of Travel Research 57(2), 232-242. DOI: https://doi.org/10.1177/0047287516688321

Kallbekken, S. \& Saelen, H. (2013) 'Nudging' hotel guests to reduce food waste as a win-win environmental measure. Economics Letters 119(3), 325-327. DOI: https://doi.org/10.1016/j.econlet.2013.03.019

Kearney, J. (2010) Food consumption trends and drivers. Philosophical Transactions of the Royal Society of London B: Biological Sciences 365(1554), 2793-2807. DOI: https://doi.org/10.1098/rstb.2010.0149

Lehner, M., Mont, O. \& Heiskanen, E. (2016) Nudging - a promising tool for sustainable consumption behaviour? Journal of Cleaner Production 134(Part A), 166-177. DOI: https://doi.org/10.1016/j. jclepro.2015.11.086

Lund-Durlacher, D., Fritz, K. \& Antonschmidt, H. (2016) Endbericht zum Futouris-Branchenprojekt „Nachhaltige Ernährung im Urlaub“. Futouris e. V., Berlin, Deutschland. Online unter: https:// www.modul.ac.at/uploads/files/user_upload/Sustainable_food_report.pdf (letzter Zugriff: 21.04.2020).

Martin-Rios, C., Demen-Meier, C., Gössling, S. \& Cornuz, C. (2018) Food waste management innovations in the foodservice industry. Waste Management 79, 196-206. DOI: https://doi.org/10.1016/j. wasman.2018.07.033

McMichael, A.J., Powles, J.W. \& Butler, C.D. (2007) Food, livestock production, energy, climate change, and health. Lancet 370(9594), 1253-1263. https://doi.org/10.1016/S0140-6736(07)61256-2

Meier, T. \& Christen, O. (2013) Environmental impacts of dietary recommendations and dietary styles: Germany as an example. Environmental Science \& Technology 47(2), 877-888. DOI: https://doi. org/10.1021/es302152v

Meinungsraum (2018) Eigenstudie Veganer/Vegetarier. meinungsraum.at Online MarktforschungsgmbH, Wien, Österreich. Online unter: https://www.businessart.at/images/doku/meinungsraumstudie_4955_eigenstudie_vegetarier_veganer.pdf (letzter Zugriff: 14.05.2019).

Ministerium für Umwelt, Landwirtschaft, Ernährung, Weinbau und Forsten Rheinland-Pfalz (2015) Nachhaltige Ernährung - was unser Essen mit Klimaschutz und Welternährung zu tun hat. Ministerium für Umwelt, Landwirtschaft, Ernährung, Weinbau und Forsten Rheinland-Pfalz, Mainz, Deutschland. Online unter: https://mueef. rlp.de/fileadmin/mulewf/Publikationen/Nachhaltige_Ernaehrung RLP_16.09.2015.pdf (letzter Zugriff: 21.04.2020).

Obersteiner, G., Sacher, C. \& Urbanova, G. (2019) Improve your loss ratio and \#reducefoodwaste-Guideline for the Food Service sector. STREFOWA - Strategies to Reduce Food Waste in Central Europe (ein Projekt des Programms Interreg CENTRAL EUROPE). Online unter: http://www.reducefoodwaste.eu/uploads/5/8/6/4/58648241/ handbook_strefowa_outcomes.pdf (letzter Zugriff: 03.06.2020).

Österreich Werbung (2019) T-Mona Kurzinfo Wein- und Kulinarikurlauber in Österreich. T-Mona-Urlauberbefragung 2017/18. Zur Verfügung gestellt von Österreich Werbung, Wien, Österreich. Online unter: https://www.austriatourism.com/tourismusforschung/ 
studien-und-berichte/wein-und-kulinarikurlauber-in-oesterreichgesamtjahr-201718/ (letzter Zugriff: 06.05.2020).

Österreichische Hagelversicherung (2017) Extreme Wettervielfalt. Österreichische Hagelversicherung VVaG, Wien, Österreich. Online unter: https://www.hagel.at/presseaussendungen/extreme-wettervielfalt-2017-250-mill-euro-gesamtschaden-der-landwirtschaft/ (letzter Zugriff: 03.06.2020).

Papargyropoulou, E., Lozano, R., Steinberger, J., Wright, N. \& bin Ujang, Z. (2014) The food waste hierarchy as a framework for the management of food surplus and food waste. Journal of Cleaner Production 76(1), 106-115. DOI: https://doi.org/10.1016/j.jclepro.2014.04.020

Poore, J. \& Nemecek, T. (2018) Reducing food's environmental impacts through producers and consumers. Science 360(6392), 987-992. DOI: https://doi.org/10.1126/science.aaq0216

ProVeg International (2019) Vegan-Trend: Zahlen und Fakten zum Veggie-Markt. ProVeg e. V., Berlin, Deutschland. Online unter: https:// proveg.com/de/vegan-trend-zahlen-und-fakten-zum-veggie-markt/ (letzter Zugriff: 14.05.2019).

Reganold, J.P. \& Wachter, J.M. (2016) Organic agriculture in the twenty-first century. Nature Plants 2(2), 15221. DOI: https://doi. org/10.1038/nplants.2015.221

Rützler, H. \& Reiter, W. (2018) Food Report 2019. Zukunftsinstitut $\mathrm{GmbH}$, Frankfurt am Main, Deutschland. Online unter: https:// www.zukunftsinstitut.de/artikel/food/trend-update-food-beveragebranche-2019/ (letzter Zugriff: 14.05.2019).

Scherhaufer, S., Moates, G., Hartikainen, H., Waldron, K. \& Obersteiner, G. (2018) Environmental impacts of food waste in Europe. Waste Management 77, 98-113. DOI: https://doi.org/10.1016/j. wasman.2018.04.038

Schmidinger, K. \& Stehfest, E. (2012) Including $\mathrm{CO}_{2}$ implications of land occupation in LCAs - method and example for livestock products. International Journal of Life Cycle Assessment 17, 962-972. DOI: https://doi.org/10.1007/s11367-012-0434-7

Schodl, B. (2017) Statusbericht zu den $\mathrm{CO}_{2}$-Emissionen neu zugelassener Pkw in Österreich im Jahr 2016. Bundesministerium für Nachhaltigkeit und Tourismus (BMNT), Wien, Österreich. Online unter: https://www.bmnt.gv.at/dam/jcr:a0918ad8-4cba-4840-9f817efe00578e6c/CO2-Monitoring Pkw\%202017\%20web.pdf (letzter Zugriff: 14.05.2019).

Statista (2017) Anzahl der veganen Gastronomiebetriebe in großen und mittelgroßen Städten in Deutschland von 2013 bis 2017. Statista $\mathrm{GmbH}$, Hamburg, Deutschland. Online unter: https://de.statista.com/ statistik/daten/studie/381076/umfrage/anzahl-veganer-gastronomiebetriebe-in-deutschland/ (letzter Zugriff: 04.11.2019).

Statistik Austria (2019a) Ankünfte und Nächtigungen nach Herkunftsländern, Kalenderjahr 2018. Bundesanstalt Statistik Österreich, Wien, Österreich. Online unter: https://www.statistik.at/web_de/ statistiken/wirtschaft/tourismus/beherbergung/ankuenfte_naechtigungen/index.html (letzter Zugriff: 14.05.2019).

Statistik Austria (2019b) Durchschnittlicher steuerbarer Umsatz 2016je Einwohner in der Wirtschaftsabteilung „Gastronomie“ nach Politischen Bezirken. In: Statistik Austria (Hrsg.) Statistik der Umsatzsteuer 2016, S. 57. Bundesanstalt Statistik Österreich, Wien, Österreich. Online unter: http://www.statistik.at/web de/services/ publikationen/19/index.html?includePage $=$ detailedView\&sectionName $=\%$ C $3 \% 96$ ffentliche + Finanzen $\% 2 C+$ Steuern $\&$ pubId $=575$ (letzter Zugriff: 14.05.2019).

Stenmarck, Å., Jensen, C., Quested, T. \& Moates, G. (2016) Estimates of European food waste levels. IVL Swedish Environmental Research Institute, Stockholm, Schweden. Online unter: https://www.eu-fusions.org/phocadownload/Publications/Estimates\%20of\%20European\%20food\%20waste\%20levels.pdf (letzter Zugriff: 03.06.2020).

Tom, M.S., Fischbeck, P.S. \& Hendrickson, C.T. (2016) Energy use, blue water footprint, and greenhouse gas emissions for current food consumption patterns and dietary recommendations in the US.
Environment Systems and Decisions 36, 92-103. DOI: https://doi. org/10.1007/s10669-015-9577-y

Umweltbundesamt (2018) Klimaschutzbericht 2018. Umweltbundesamt $\mathrm{GmbH}$, Wien, Österreich. Online unter: https://www.umweltbundesamt.at/fileadmin/site/publikationen/REP0660.pdf (letzter Zugriff: 30.04.2020).

UN (2017) World population projected to reach 9.8 billion in 2050, and 11.2 billion in 2100. United Nations, Department of Economic and Social Affairs (UN DESA), New York, NY, USA. Online unter: https://www.un.org/development/desa/en/news/population/worldpopulation-prospects-2017.html (letzter Zugriff: 14.05.2019).

United Against Waste (2015) Vermeidung von Lebensmittelabfall in Gastronomie, Beherbergung und Großküchen: Endbericht, Mai 2015. Initiative United Against Waste, Wien, Österreich. Online unter: https://united-against-waste.at/wp-content/uploads/2015/05/ Endbericht_UAW_ABF_tatwort_final_ARA.pdf?eb6772 (letzter Zugriff: 14.05.2019).

United Against Waste (2016) United Against Waste:Rückblick, Erhebungsergebnisse \& Ausblick. Fachmediengespräch vom 21.01.2016. Initiative United Against Waste, Wien, Österreich. Online unter: https://united-against-waste.at/wp-content/uploads/2016/01/2016 Pr\%C3\%A4sentation-UAW-Abschluss-PK.pdf (letzter Zugriff: 14.05.2019).

United Against Waste (2018) Lebensmittelabfälle vermeiden, Kosten sparen \& Umwelt schützen: Einsparstipps \& Serviceangebote für Gastronomie und Hotellerie. Initiative United Against Waste, Wien, Österreich. Online unter: https://united-against-waste.at/wp-content/ uploads/2015/05/UAW_Gastro_LR.pdf?fa6be0 (letzter Zugriff: 14.05.2019).

UNWTO (2018) UNWTO Tourism Highlights: 2018 Edition. World Tourism Organization (UNWTO), Madrid, Spanien. Online unter: https:// doi.org/10.18111/9789284419876 (letzter Zugriff: 21.04.2020).

Visschers, V. H. \& Siegrist, M. (2015) Does better for the environment mean less tasty? Offering more climate-friendly meals is good for the environment and customer satisfaction. Appetite 95(1), 475-483. DOI: https://doi.org/10.1016/j.appet.2015.08.013

Vitousek, P.M., Mooney, H.A., Lubchenco, J. \& Melillo, J.M. (1997) Human domination of Earth's ecosystems. Science 277(5325), 494 499. DOI: https://doi.org/10.1126/science.277.5325.494

VVÖ (2016) Naturkatastrophen in Österreich: Jeder ist betroffen! Verband der Versicherungsunternehmen Österreichs, Wien, Österreich. Online unter: https://www.vvo.at/vvo/vvo.nsf/sysPages/4C443C9E5 BA59528C125804400317CCB (letzter Zugriff: 14.05.2019).

Wansink, B. \& van Ittersum, K. (2003) Bottoms up! The influence of elongation on pouring and consumption volume. Journal of Consumer Research 30(3), 455-463. DOI: https://doi.org/10.1086/378621

Westhoek H., Lesschen, J.P., Rood, T., Wagner, S., De Marco, A., Murphy-Bokern, D., Leip, A., van Grinsven, H., Sutton, M. A. \& Oenema, O. (2014) Food choices, health and environment: effects of cutting Europe's meat and dairy intake. Global Environmental Change 26, 196-205. DOI: https://doi.org/10.1016/j.gloenvcha.2014.02.004

Wheeler, T. \& von Braun, J. (2013) Climate change impacts on global food security. Science 341(6145), 508-513. DOI: https://doi. org/10.1126/science. 1239402

WIFI (2002) Energieeffizienz Gastronomie. WIFI Unternehmerservice der WKÖ, Wien, Österreich. Online unter: http://www.win.steiermark.at/cms/dokumente/11263992 52486039/06e4795f/Energieeffizienz\%20Gastronomie1.pdf (letzter Zugriff: 14.05.2019).

WKO (2020) Gastronomie Branchendaten. Wirtschaftskammer Österreich (WKO), Abteilung für Statistik, Wien, Österreich. Online unter: http://wko.at/statistik/BranchenFV/B_601.pdf(letzter Zugriff: 21.04.2020).

Zollitsch, W., Winkler, C., Waiblinger, S. \& Haslberger, A. (2007) Sustainable food production and ethics. Wageningen Academic Publishers, Wageningen, Niederlande. DOI: https://doi.org/10.3920/97890-8686-616-8 
Open Access Dieses Buch wird unter der Creative Commons Namensnennung 4.0 International Lizenz (http://creativecommons.org/licenses/by/4.0/deed.de) veröffentlicht, welche die Nutzung, Vervielfältigung, Bearbeitung, Verbreitung und Wiedergabe in jeglichem Medium und Format erlaubt, sofern Sie den/die ursprünglichen Autor(en) und die Quelle ordnungsgemäß nennen, einen Link zur Creative Commons Lizenz beifügen und angeben, ob Änderungen vorgenommen wurden.

Die in diesem Buch enthaltenen Bilder und sonstiges Drittmaterial unterliegen ebenfalls der genannten Creative Commons Lizenz, sofern sich aus der Abbildungslegende nichts anderes ergibt. Sofern das betreffende Material nicht unter der genannten Creative Commons Lizenz steht und die betreffende Handlung nicht nach gesetzlichen Vorschriften erlaubt ist, ist für die oben aufgeführten Weiterverwendungen des Materials die Einwilligung des jeweiligen Rechteinhabers einzuholen. 